\title{
Effects of a 4-week Wheat Bran Diet Combined with Weight Self-monitoring on Visceral Fat and Body Weight Among Overweight Individuals
}

\author{
Nami Yamanaka ${ }^{1}$, Shinichiro Saito ${ }^{1,}$, Kazuya Kozuma $^{2}$, Noriko Osaki ${ }^{2}$, Yoshihisa Katsuragi ${ }^{2}$ \\ ${ }^{1}$ Biological Science Research Laboratories, Kao Corporation, Tokyo, Japan \\ ${ }^{2}$ Health and Wellness Research Laboratories, Kao Corporation, Tokyo, Japan
}

Email address:

saito.shinichiro@kao.com (S. Saito)

${ }^{*}$ Corresponding author

To cite this article:

Nami Yamanaka, Shinichiro Saito, Kazuya Kouzuma, Noriko Osaki, Yoshihisa Katsuragi. Effects of a 4-week Wheat Bran Diet Combined with Weight Self-monitoring on Visceral Fat and Body Weight Among Overweight Individuals. International Journal of Nutrition and Food Sciences. Vol. 9, No. 1, 2020, pp. 10-15. doi: 10.11648/j.ijnfs.20200901.13

Received: January 16, 2020; Accepted: February 7, 2020; Published: March 10, 2020

\begin{abstract}
Background: Animal studies have shown that wheat bran (WB), which is rich in dietary fiber, has beneficial effects against postprandial hyperglycemia and fatty liver. The present study thus investigated the effects of a WB diet combined with body weight self-monitoring on visceral fat area (VFA) and body weight among overweight participants. Method: This single-blind randomized controlled trial was conducted in adults with a body mass index of 23 to $<30 \mathrm{~kg} / \mathrm{m}^{2}$. After screening, 44 participants were randomly allocated to either the combination or control group ( $\mathrm{n}=22 /$ group). The combination group was instructed to consume $8 \mathrm{~g}$ /day of WB (1.6 g/day as arabinoxylan) with their regular diet and monitor their body weight daily for 4 weeks, whereas the control group was instructed to maintain their regular diet and monitor their body weight daily for 4 weeks. Results: Although both groups exhibited a decrease in VFA, i.e., the primary outcome measure, the combination group exhibited a greater reduction therein compared with the control group with a significant treatment by time interaction. Moreover, the combination group exhibited a significantly greater decrease in body weight, i.e., the secondary outcome measure, after 4 weeks than the control group. Conclusion: Incorporating WB into the daily diet together with weight self-monitoring therapy can be beneficial for reducing VFA and body weight.
\end{abstract}

Keywords: Arabinoxylan, Human, Self-monitoring, Visceral Fat, Wheat Bran

\section{Introduction}

Over the last two decades, a dramatic increase in obesity rates has been observed in the global population with as many as 711.4 million obese individuals in 2015 [1]. Studies have shown that visceral fat accumulation is more strongly associated with metabolic risk factors than subcutaneous fat accumulation [2] while also being associated with insulin resistance, hypertension, and dyslipidemia [3]. In addition, visceral adipose tissue has been associated with cardiovascular disease and cancer [4], suggesting that visceral fat area (VFA) reduction could potentially prevent the development of such diseases. Body weight self-monitoring has been considered a first-line therapy for obesity that affects patient behavior by improving motivation, which results in successful weight loss [5-7]. Wheat bran (WB) is a hulled constituent of whole grain that is rich in dietary fiber. Accordingly, the dietary fiber in wheat bran is mostly insoluble, with arabinoxylan accounting for approximately $50 \%$ of the total dietary fiber [8]. Studies have shown that wheat bran and arabinoxylan decrease blood glucose concentrations [9-13] and liver triglycerides in mice [14] and the risk for coronary heart disease in humans [15, 16]. Moreover, mice treated with arabinoxylan exhibited weight loss and reduced VFA $[17,18]$. To gain optimistic result following consumption of dietary fiber, high compliance for its repeated consumption would be required. In general, however, maintaining its motivation for a long-term is thought to be difficult due to individuals' preferences for the taste and dietary habits. Therefore, we 
hypothesized that maintaining motivation for its repeated consumption is a key factor to gain successful weight loss. We also hypothesized that self-monitoring of weight could lead to a visualized achievement, and it may be expected participants' high compliance of consumption rate of the test diet. Thus, the present study investigated the effects of a WB diet combined with weight self-monitoring on VFA and body weight among overweight individuals.

\section{Materials and Methods}

\subsection{Management}

The study protocol was registered with the University Hospital Medical Information Network (UMIN) Center (UMIN-CTR, http://www.umin.ac.jp/ctr/index-j.htm) before enrolling the first participant (UMIN test ID: UMIN000025279). The study was conducted at Higashi Shinjuku Clinic (Tokyo, Japan) under the supervision of the physician in charge, whereas the operation and management were outsourced to a contract research organization, New Drug Research Center Co., Ltd. (Tokyo, Japan).

\subsection{Study Design}

This study was a single-blind randomized controlled trial. Statistical analyses were conducted under blinded conditions. The control group was instructed to maintain their regular diet and monitor their body weight daily for 4 weeks. Contrarily, the combination group was instructed to monitor their body weight daily and consume $8 \mathrm{~g}$ /day of WB (1.6 g/day as arabinoxylan) in the form of a dietary bar in addition to their regular diet (breakfast, lunch, or dinner) for 4 weeks. Measurements were performed upon screening and at the 0-, 2-, and 4-week visitations. The primary outcome measure was VFA, whereas the secondary outcome measures were body weight, body mass index (BMI), and waist circumference (WC). Participants were instructed to record their diet 3 days before each measurement, and physical activity was measured using a pedometer during the study period.

\subsection{Participants}

Potential participants were recruited from volunteers living around the Tokyo metropolitan area and screened based on the inclusion and exclusion criteria. The inclusion criteria were healthy men and women between 20 and 64 years of age with a BMI of 23 to $<30 \mathrm{~kg} / \mathrm{m}^{2}$. The exclusion criteria were as follows: (1) the presence of liver, kidney, or cardiovascular disease; respiratory, endocrine, metabolic, nervous system, or cognitive disorders; diabetes mellitus; or other diseases, (2) abnormal laboratory values or physician-diagnosed disease, (3) surgery within 2 months before the trial, (4) taking medications for hyperglycemia, hyperlipidemia, or hypertension, (5) taking supplements or foods authorized by the government as specific health treatments, (6) blood donation of $200 \mathrm{~mL}$ or more within 1 month before the trial, (7) weight changes of $3 \mathrm{~kg}$ or more within 3 months before the trial, (8) allergies to any component of the test diet, (9) plans to participate in another clinical trial, and (10) the inability to provide informed consent. Power calculation was based on the results of our unpublished pilot study showing reduced VFA after WB treatment. Accordingly, a sample size of 20 participants per group was considered adequate for detecting changes in VFA with a two-tailed alpha error of 0.05 and a power of $80 \%$.

Participants who provided written informed consent were allocated to either the control or combination group using stratified block randomization with sex and VFA as stratification factors and a block size of 2. Investigators, assessors, and caregivers were blinded to the group allocation throughout the study and analyses.

\subsection{Tests Diets}

WB was purchased from Nisshin Pharma Inc. (Wheat bran DF; Tokyo, Japan). Arabinoxylan amount in wheat bran was calculated using the following equation. Arabinoxylan $=0.88$ $\times$ (arabinose + xylose $-0.7 \times$ galactose $)$. The WB-containing diet was prepared by mixing $8 \mathrm{~g}$ of WB and flour with egg, margarine, sugar, soy protein, emulsifier, sweetener, leavening agent, and starch and baking the mixture in an oven. Each diet had the following nutritional value: $1005 \mathrm{~kJ}$ of energy, $20 \%$ protein, $27 \%$ fat, $53 \%$ carbohydrates, and 4.4 $\mathrm{g}$ of dietary fiber per serving. The test diets were manufactured by Hamada Confect Co., Ltd (Hyogo, Japan).

\subsection{Measurements}

VFA was measured using a visceral fat scale (EW-FA90, Panasonic Corporation, Osaka, Japan) whose results highly correlate with those obtained using computed tomography [19]. Blood samples were analyzed by SRL Co., Ltd (Tokyo, Japan). All measurements were performed by the contract research organization members who were blinded to the experimental conditions.

\subsection{Statistical Analysis}

Analyses were conducted using SAS version 9.3 (SAS Institute Japan, Inc. Tokyo Japan) with a p-value of $<0.05$ being considered statistically significant. Data obtained from participants whose primary outcome was measured at 0 and 4 weeks without protocol violation were included for analyses. Significant differences in the primary and secondary outcomes were assessed using a linear mixed model with fixed effects for treatment and time interaction. As an exploratory analysis, between-group differences in changes from baseline to 4 weeks were assessed using Student's t-test. Non-normally distributed data were log-transformed prior to analysis. The Mann-Whitney U test was performed for data that could not be log-transformed. Incidences of adverse events were assessed using Fisher's exact test.

\section{Results}

\subsection{Participant Characteristics}

Among the 107 individuals who underwent screening, 44 
were randomly allocated to either the control or combination group ( $n=22$ /group), all of whom were analyzed for adverse events. The following participants were excluded from our analyses: one participant from the control group who did not show up for the 0-week visit, one participant from the control group who did not complete primary outcome measurements at 4 weeks due to problems with the measuring instruments, and one participant each from the control and combination groups who failed to comply with the study protocol (did not maintain habitual physical activity and diet). Thus, 19 (men/women, 15/4; age, $44 \pm 14$ years) and 21 (men/women, 16/5; age, $44 \pm 10$ years) participants in the control and combination groups were ultimately included for analyses, respectively.

\subsection{Compliance and Dietary Record}

Mean consumption rate of test diets was $99.2 \%$ (Min $86 \%-100 \%)$ in the combination group. Table 1 presents the dietary and physical activity data during the study period. Energy, protein, and fat intake did not significantly differ between both groups throughout the intervention period. However, the combination group had a significantly lower carbohydrate intake at 4 weeks and a significantly higher dietary fiber intake at 2 and 4 weeks compared with the control group, presumably due to WB loading. Moreover, the combination group took significantly fewer daily steps than the control group.

Table 1. Diet record and physical activity.

\begin{tabular}{|c|c|c|c|c|c|c|c|c|c|c|c|c|c|}
\hline & & $0 w$ & & & $2 w$ & & & $4 w$ & & & $\Delta 4 w$ & & \\
\hline Energy & control & 7401 & \pm & 1586 & 7155 & \pm & 2155 & 7653 & \pm & 2125 & 251 & \pm & 1314 \\
\hline$(\mathrm{kJ} / \mathrm{d})$ & combination & 6904 & \pm & 1812 & 6682 & \pm & 1180 & 6489 & \pm & 1607 & -414 & \pm & 1720 \\
\hline Protein & control & 65.0 & \pm & 14.1 & 64.5 & \pm & 20.1 & 69.3 & \pm & 18.4 & 4.3 & \pm & 14.1 \\
\hline$(g / d)$ & combination & 64.9 & \pm & 21.5 & 65.1 & \pm & 14.0 & 63.3 & \pm & 12.6 & -1.6 & \pm & 21.9 \\
\hline Fat & control & 56.1 & \pm & 15.5 & 55.5 & \pm & 27.5 & 59.4 & \pm & 24.8 & 3.3 & \pm & 16.6 \\
\hline$(g / d)$ & combination & 51.2 & \pm & 20.4 & 51.8 & \pm & 15.8 & 52.2 & \pm & 31.4 & 1.1 & \pm & 25.7 \\
\hline Carbohydrate & control & 241.4 & \pm & 51.1 & 227.5 & \pm & 66.8 & 241.8 & \pm & 60.8 & 0.4 & \pm & 44.4 \\
\hline Dietary fiber & control & 11.0 & \pm & 4.0 & 9.4 & \pm & 3.4 & 9.3 & \pm & 3.0 & -1.6 & \pm & 3.8 \\
\hline$(\mathrm{g} / \mathrm{d})$ & combination & 10.9 & \pm & 4.0 & $\begin{array}{l}11.9 \\
0 \sim 2 \mathrm{~W}\end{array}$ & \pm & $2.8^{*}$ & $\begin{array}{l}11.3 \\
2 \sim 4 \mathrm{w}\end{array}$ & \pm & $2.7^{*}$ & 0.4 & \pm & 3.6 \\
\hline $\begin{array}{l}\text { Physical activity } \\
\text { (steps/d) }\end{array}$ & $\begin{array}{l}\text { control } \\
\text { combination }\end{array}$ & & & & $\begin{array}{l}8739 \\
6385\end{array}$ & $\begin{array}{l} \pm \\
\pm\end{array}$ & $\begin{array}{l}2251 * * \\
2159\end{array}$ & $\begin{array}{l}8336 \\
6806\end{array}$ & $\begin{array}{l} \pm \\
\pm\end{array}$ & $\begin{array}{l}2269^{*} \\
2311\end{array}$ & & & \\
\hline
\end{tabular}

Values are mean $\pm \mathrm{SD}$. Significant difference between the groups by Student's t-test; ${ }^{*} \mathrm{p}<0.01,{ }^{*} \mathrm{p}<0.05$.

\subsection{Visceral Fat Area}

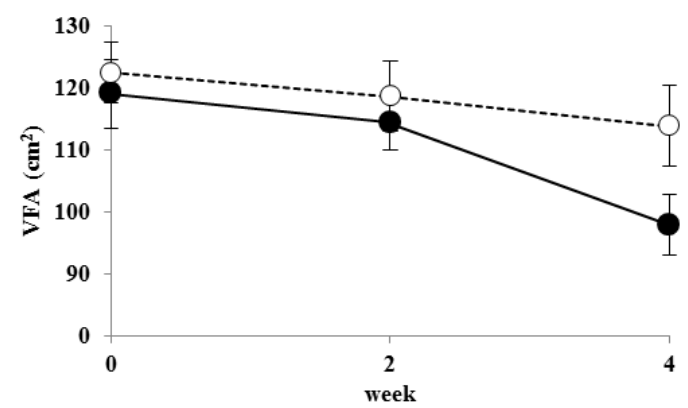

Figure 1. Visceral fat area changes in the control group $(n=19$; dotted line, open circle) and combination group ( $n=21$; solid line, closed circle) throughout the study period. Values represent mean \pm standard error. A significant treatment by time interaction was observed for visceral fat area.
A significant treatment by time interaction was observed for VFA (Figure 1; $\mathrm{P}=0.017$ ). The reduction in VFA at week 4 was $-9 \pm 19 \mathrm{~cm}^{2}($ mean $\pm \mathrm{SD})$ in the control group and -21 $\pm 18 \mathrm{~cm}^{2}$ in the combination group.

\subsection{Body Weight, Body Mass Index and Waist Circumference}

No significant treatment by time interaction was observed for any of the secondary outcomes. The exploratory assessment regarding changes from baseline to 4 weeks revealed that the combination group had a significantly lower body weight and $\mathrm{BMI}$, but not $\mathrm{WC}$, than the control group (Table 2).

Table 2. Change in VFA, body weight, BMI and WC.

\begin{tabular}{|c|c|c|c|c|c|c|c|c|c|c|c|c|c|}
\hline & & $\mathbf{0 w}$ & & & $2 w$ & & & $4 w$ & & & $\Delta 4 w$ & & \\
\hline VFA & control & 122 & \pm & 21.7 & 119 & \pm & 24.6 & 114 & \pm & 28.4 & -9 & \pm & 18.8 \\
\hline$\left(\mathrm{cm}^{2}\right)$ & combination & 119 & \pm & 25.3 & 114 & \pm & 20.4 & 98 & \pm & $22 \#$ & -21 & \pm & $18 *$ \\
\hline Body weight & control & 71.8 & \pm & 6.9 & 71.6 & \pm & 6.5 & 71.3 & \pm & 6.5 & -0.5 & \pm & 0.7 \\
\hline$(\mathrm{kg})$ & combination & 69.8 & \pm & 9.1 & 69.2 & \pm & 9.0 & 68.4 & \pm & 8.8 & -1.4 & \pm & $1.1^{*}$ \\
\hline BMI & control & 25.4 & \pm & 1.7 & 25.3 & \pm & 1.7 & 25.2 & \pm & 1.6 & -0.2 & \pm & 0.3 \\
\hline$\left(\mathrm{kg} / \mathrm{m}^{2}\right)$ & combination & 27.5 & \pm & 1.8 & 25.5 & \pm & 1.8 & 25.3 & \pm & 1.8 & -0.4 & \pm & $0.4^{*}$ \\
\hline WC & control & 91.8 & \pm & 4.4 & 91.1 & \pm & 4.4 & 90.6 & \pm & 4.5 & -1.2 & \pm & 1.9 \\
\hline
\end{tabular}

Values are mean $\pm \mathrm{SD}$.

The treatment by time interaction from 0 to $4 \mathrm{wk}$ were assessed by linear mixed model; $\#<0.05$

As exploration data analyses, significant differences between the groups were assessed changes from the baseline to 4 -wk by Student's t-test; $* 0.05$ 


\subsection{Other Parameters}

Both groups exhibited no significant differences in systolic and diastolic blood pressure, triglycerides, glucose, insulin, and total ketone bodies (Table 3).

Table 3. Change in other parameter.

\begin{tabular}{|c|c|c|c|c|c|c|c|c|c|c|c|c|c|}
\hline & group & & $0 w$ & & & $2 w$ & & & $4 w$ & & $\Delta 4 w$ & & \\
\hline SBP & control & 121 & \pm & 9 & 124 & \pm & 15 & 118 & \pm & 13 & -2 & \pm & 11 \\
\hline (mmHg) & combination & 118 & \pm & 13 & 118 & \pm & 10 & 119 & \pm & 13 & 1 & \pm & 8 \\
\hline DBP & control & 77 & \pm & 10 & 77 & \pm & 12 & 76 & \pm & 10 & -1 & \pm & 6 \\
\hline TG & control & 1.22 & \pm & 0.73 & 1.15 & \pm & 0.53 & 1.08 & \pm & 0.49 & -0.14 & \pm & 0.51 \\
\hline$(\mathrm{mmol} / \mathrm{L})$ & combination & 1.14 & \pm & 0.56 & 1.00 & \pm & 0.52 & 1.07 & \pm & 0.76 & -0.07 & \pm & 0.72 \\
\hline Glucose & control & 5.06 & \pm & 0.65 & 5.01 & \pm & 0.59 & 5.09 & \pm & 0.47 & 0.03 & \pm & 0.37 \\
\hline Insulin & control & 48.7 & \pm & 18.3 & 48.0 & \pm & 19.9 & 41.9 & \pm & 16.5 & -6.8 & \pm & 19.2 \\
\hline$(\mathrm{pmol} / \mathrm{L})$ & combination & 55.7 & \pm & 35.9 & 46.3 & \pm & 18.0 & 51.8 & \pm & 36.2 & -4.0 & \pm & 32.6 \\
\hline TKB & control & 74.6 & \pm & 61.5 & 116.6 & \pm & 136.2 & 65.2 & \pm & 63.6 & -9.5 & \pm & 70.7 \\
\hline$(\mu \mathrm{mol} / \mathrm{L})$ & combination & 108.0 & \pm & 126.3 & 86.7 & \pm & 75.2 & 116.1 & \pm & 108.7 & 8.2 & \pm & 159.2 \\
\hline
\end{tabular}

Values are mean $\pm \mathrm{SD}$. Significant difference between the groups by Student's t-test; $* \mathrm{p}<0.05$.

Abbreviations: SBP, systolic blood pressure; DBP, diastolic blood pressure; TG, triglycerides

\subsection{Safety}

Adverse events occurred in three participants from the control group (cold symptoms, sore throat, and moderate backache) and three from the combination group (cold symptoms, headache, and stomatitis). Fisher's exact test revealed no significant differences in the incidence of adverse events between both groups. Furthermore, the observed symptoms were mild to moderate, and the physician in charge determined no cause-and-effect relationship between the test diets and adverse events in the combination group.

\section{Discussion}

The present study compared the effects of a WB diet combined with body weight self-monitoring with that of weight self-monitoring alone on VFA among overweight individuals. Accordingly, our results revealed that the combination of a WB diet and weight self-monitoring effectively reduced VFA and body weight. Previous reports [5-7] have shown that weight self-monitoring alone can be effective in reducing body weight. Indeed, the present study revealed that the control group exhibited a significant decrease in VFA and body weight from baseline. In our unpublished pilot study involving 18 participants with a BMI similar to that of the participants included in this study, WB diet alone reduced VFA by $6 \pm 11 \mathrm{~cm}^{2}$, whereas combining WB diet with weight self-monitoring, as done herein, reduced the same by $21 \pm 12 \mathrm{~cm}^{2}$. Given that the magnitude of reduction attained by combining both treatments was greater than the sum of each treatment despite decreased activity in the combination group (Table 1), WB has been presumed to have the potential to enhance the effects of weight self-monitoring. Additionally, despite the additional energy value of the WB diets $(1005 \mathrm{~kJ})$ consumed by the combination group in addition to their regular diet, their total energy consumption was reduced by $418 \mathrm{~kJ}$ at 4 weeks. Presumably, this decrease could be associated with a feeling of fullness induced by the abundance of fiber in the WB, which may have promoted a greater reduction in VFA in the combination group.

A previous study of mice found that a single oral ingestion of WB decreases postprandial blood levels of glucose-dependent insulinotropic polypeptides and increases postprandial fat oxidation [20]. The extrapancreatic action of glucose-dependent insulinotropic polypeptides is to stimulate fat accumulation in the adipose tissue [21]. Together with increased fat oxidation, upregulated fat metabolism has been one of the main mechanisms of action underlying our present observations.

Recent studies suggest that gut microbiota have the potential to regulate human body weight $[22,23]$. The composition or variation of gut microbiota can be modified by altering one's regular diet, particularly the consumption of fermentable food constituents such as oligosaccharides and fiber. Accordingly, animal models have shown that WB and arabinoxylan, the main fiber in $\mathrm{WB}$, change gut microbiota and decrease visceral adipose tissue in animal models [17, 18]. Moreover, digestion of WB produces short-chain fatty acids, such as acetic acid, butyric acid, and propionic acid [24], probably as fermentation byproducts produced by intestinal microbiota. Butyric acid improves insulin sensitivity, increases energy expenditure, and suppresses weight gain [25], suggesting that microbiota activities could be potentially involved in the present findings. Nonetheless, further studies are necessary to clarify the association between gut microbiota and the effects of WB on human obesity.

$\mathrm{Lu}$ et al. [9] reported that arabinoxylan fiber improves fasting plasma glucose levels in type 2 diabetes. Although Garcia et al. [10] reported that administering $15 \mathrm{~g} /$ day of arabinoxylan to participants with impaired glucose tolerance improved fasting serum glucose, the present study found no 
significant differences in blood glucose between both groups. This discrepancy may be due to our study's inclusion of overweight but healthy participants who had normal blood glucose levels and/or underwent a relatively short-term intervention (4 weeks).

The combination of a WB diet and weight self-monitoring showed decrease in VFA by a relatively short-term intervention and it may be able to give incentive to manage weight control in people with overweight and obesity. Moreover, dietary pattern with high dietary fiber is supposed to be beneficial to reduce risk of rebound and future risk of metabolic syndrome related diseases. Thus, even after weight loss, the combination of a WB diet and weight self-monitoring is desired to be continued to maintain healthy body weight. Therefore, it is required to be demonstrated a further study with a longer period of intervention.

\section{Conclusion}

The present study showed that incorporating WB into the regular diet, in combination with body weight self-monitoring, can be beneficial for reducing VFA without any apparent side effects. In addition, WB diet combined with body weight self-monitoring may be available as a program for a first-line therapy in people with obesity.

\section{Limitations}

Although measurements and analyses were conducted under blinded conditions until completed, participants were aware of their own group and thereby may have been biased, which could have affected our results.

\section{List of Abbreviations}

BMI: body mass index; VFA: visceral fat area; WB: wheat bran; WC: waist circumference

\section{Acknowledgements}

We deeply appreciate Mr. Daisuke Shiiba, Mr. Tomohiro Nakagawa, Mr. Shohei Takahashi, and Mr. Hiroshi Aoyama of the Health and Wellness Research Laboratories at Kao Corporation for preparing the test diets. We deeply appreciate Dr. Haruo Nakamura of Mitsukoshi Health and Welfare Foundation for instruction and advice regarding the study design, protocol, and discussion. We deeply appreciate Dr. Haruna Miura of Higashi Shinjyuku Clinic for supervising the study.

\section{Statement of Ethics}

This study was conducted in accordance with the Declaration of Helsinki (2013) and was approved by the Miyawaki Orthopedic Hospital Institutional Review Board on 28 November 2016. After receiving a full explanation of the study, all participants provided written informed consent.

\section{Authors' Contributions}

NY was responsible for conceiving and designing the study and drafting the manuscript. SS was responsible for conceiving and designing the study and revising the manuscript. KK provided advice regarding the study design and data analysis. NO and KY read and approved the final manuscript.

\section{References}

[1] GBD 2015 Obesity Collaborators, Afshin A, Forouzanfar MH, Reitsma MB, Sur P, Estep K, et al (2017). Health effects of overweight and obesity in 195 countries over 25 years. N Engl J Med 377 (1): 13-27.

[2] Fox CS, Massaro JM, Hoffmann U, Pou KM, Maurovich-Horvat P, Liu CY, et al (2007). Abdominal visceral and subcutaneous adipose tissue compartments: association with metabolic risk factors in the Framingham Heart Study. Circulation 116 (1): 39-48.

[3] Matsuzawa Y (2014). Obesity and metabolic syndrome: the contribution of visceral fat and adiponectin. Diabetes Management 4 (4): 391-401.

[4] Britton KA, Massaro JM, Murabito JM, Kreger BE, Hoffmann U, Fox CS (2013). Body fat distribution, incident cardiovascular disease, cancer, and all-cause mortality. J Am Coll Cardiol 62 (10): 921-25.

[5] Zheng Y, Burke LE, Danford CA, Ewing LJ, Terry MA, Sereika SM (2016). Patterns of self-weighing behavior and weight change in a weight loss trial. Int J Obes 40 (9): 1392-6.

[6] VanWormer JJ, Martinez AM, Martinson BC, Crain AL, Benson GA, Cosentino DL, et al (2009) Self-weighing promotes weight loss for obese adults. Am J Prev Med 36 (1): $70-3$.

[7] Butryn ML, Phelan S, Hill JO, Wing RR (2007). Consistent self-monitoring of weight: A key component of successful weight loss maintenance. Obesity 15 (12): 3091-6.

[8] Stevenson L, Phillips F, O'Sullivan K, Walton J (2012). Wheat bran: its composition and benefits to health, a European perspective. Int J Food Sci Nutr 63 (8): 1001-13.

[9] Lu ZX, Walker KZ, Muir JG, O'Dea K (2004). Arabinoxylan fibre improves metabolic control in people with Type II diabetes. Eur J Clin Nutr 58 (4): 621-8.

[10] Garcia AL, Steiniger J, Reich SC, Weickert MO, Harsch I, Machowetz A, et al (2006). Arabinoxylan fibre consumption improved glucose metabolism, but did not affect serum adipokines in subjects with impaired glucose tolerance. Horm Metab Res 38 (11): 761-6.

[11] Lu ZX, Walker KZ, Muir JG, Mascara T, O'Dea K (2000). Arabinoxylan fiber, a byproduct of wheat flour processing, reduces the postprandial glucose response in normoglycemic subjects. Am J Clin Nutr 71 (5): 1123-8.

[12] Hartvigsen ML, Lærke HN, Overgaard A, Holst JJ, Bach Knudsen KE, Hermansen K (2014). Postprandial effects of test meals including concentrated arabinoxylan and whole grain rye in subjects with the metabolic syndrome: a randomised study. Eur J Clin Nutr 68 (5): 567-74. 
[13] Numan Ahmad M, Rabah Takruri H (2015). The effect of dietary wheat bran on sucrose-induced changes of serum glucose and lipids in rats. Nutr Hosp 32 (4): 1636-44.

[14] Han S, Jiao J, Zhang W, Xu J, Wan Z, Zhang W, et al (2015). Dietary fiber prevents obesity-related liver lipotoxicity by modulating sterol-regulatory element binding protein pathway in C57BL/6J mice fed a high-fat/cholesterol diet. Sci Rep 5: 15256.

[15] Jensen MK, Koh-Banerjee P, Hu FB, Franz M, Sampson L, Gronbaek M, et al (2004). Intakes of whole grains, bran, and germ and the risk of coronary heart disease in men. Am J Clin Nutr 80 (6): 1492-9.

[16] He M, van Dam RM, Rimm E, Hu FB, Qi L (2010). Whole-grain, cereal fiber, bran, and germ intake and the risks of all-cause and cardiovascular disease-specific mortality among women with type 2 diabetes mellitus. Circulation 121 (20): $2162-8$.

[17] Neyrinck AM, Possemiers S, Druart C, Van de Wiele T, De Backer F, Cani PD, et al (2011). Prebiotic effects of wheat arabinoxylan related to the increase in bifidobacteria, Roseburia and Bacteroides/Prevotella in diet-induced obese mice. PLoS One 6 (6): e20944.

[18] Neyrinck AM, Van Hée VF, Piront N, De Backer F, Toussaint O, Cani PD, et al (2012). Wheat-derived arabinoxylan oligosaccharides with prebiotic effect increase satietogenic gut peptides and reduce metabolic endotoxemia in diet-induced obese mice. Nutr Diabetes 2: e28.
[19] Ryo M, Maeda K, Onda T, Katashima M, Okumiya A, Nishida $\mathrm{M}$, et al (2005). A new simple method for the measurement of visceral fat accumulation by bioelectrical impedance. Diabetes Care 28 (2): 451-3.

[20] Hosoda Y, Okahara F, Mori T, Deguchi J, Ota N, Osaki N, et al (2017). Dietary steamed wheat bran increases postprandial fat oxidation in association with a reduced blood glucose-dependent insulinotropic polypeptide response in mice. Food Nutr Res 23; 61 (1): 1361778.

[21] Seino Y, Fukushima M, Yabe D (2010). GIP and GLP-1, the two incretin hormones: Similarities and differences. J Diabetes Investig. 2010 Apr 22; 1 (1-2): 8-23.

[22] Ley RE, Turnbaugh PJ, Klein S, Gordon JI (2006). Microbial ecology: human gut microbes associated with obesity. Nature 21; 444 (7122): 1022-3.

[23] Duncan SH, Lobley GE, Holtrop G, Ince J, Johnstone AM, Louis P, et al (2008). Human colonic microbiota associated with diet, obesity and weight loss. Int J Obes (Lond) 32 (11): $1720-4$.

[24] Anson NM, Havenaar R, Vaes W, Coulier L, Venema K, Selinheimo E, et al. (2011). Effect of bioprocessing of wheat bran in wholemeal wheat breads on the colonic SCFA production in vitro and postprandial plasma concentrations in men. Food Chem 15; 128 (2): 404-9.

[25] Gao Z, Yin J, Zhang J, Ward RE, Martin RJ, Lefevre M, et al (2009) Butyrate improves insulin sensitivity and increases energy expenditure in mice. Diabetes 58 (7): 1509-17. 